\title{
Cardiopatías congénitas asociadas a los síndromes cromosómicos más prevalentes: revisión de la literatura
}

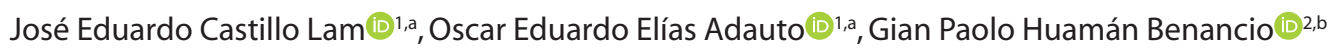

Recibido: 11 de septiembre de 2021. Aceptado: 25 de septiembre de 2021

Filiación de los autores

Universidad Peruana Cayetano Heredia. Lima, Perú.

Instituto Nacional Cardiovascular INCOR. Lima, Perú.

Estudiante de medicina humana.

Médico cardiólogo pediatra.

\section{*Correspondencia}

José Eduardo Castillo Lam

Jirón Leoncio Prado 363 Dpto. 103.

Magdalena, Lima, Perú

+51952687872

Correo

jose.castillo.lam@upch.pe

Financiamiento

Autofinanciado

Conflictos de interés

No se declaran conflictos de interés.

Citar como:

Castillo Lam JE, Elías Adauto OE, Human Benancio GP. Cardiopatías congénitas asociadas a los síndromes cromosómicos más prevalentes: revisión de la literatura. Arch Peru Cardiol Cir Cardiovasc. 2021:2(3):187195. doi: $10.47487 / a p c y c c v . v 2 i 3.155$

\section{RESUMEN}

Los síndromes cromosómicos más frecuentes (Down, Patau, Edwards, Turner y Williams) afectan de diversas formas a la población pediátrica, y las cardiopatías congénitas asociadas explican la compleja calidad de vida que padecen; sin embargo, no se cuenta con muchos estudios que hagan una revisión acerca de las principales anomalías cardiacas en estos síndromes, y los que existen son publicaciones de décadas pasadas. Se hizo una revisión en bases de datos como MEDLINE, LILACS, SCIELO y Google Scholar, para seleccionar la mejor evidencia posible; cada síndrome cromosómico se investigó en relación con cardiopatías congénitas, por separado, y se organizaron cinco grupos de búsqueda. El artículo muestra las características de cada una de las cardiopatías asociadas que fueron descritas en los estudios revisados, información relevante de cada estudio (autor, fecha de publicación, país y población estudiada), además de una breve descripción que consigna la frecuencia de la cardiopatía y su mortalidad. Se contrastaron los resultados descritos en esta revisión, con literatura previa ya existente con la finalidad de verificar la existencia de correspondencia entre las frecuencias reportadas. Las cardiopatías congénitas más frecuentes fueron: defecto septal atrioventricular (DSAV), comunicación interventricular (CIV), comunicación interauricular (CIA) y persistencia de conducto arterioso (PCA) en el síndrome Down; PCA, CIA y CIV en el síndrome Patau; DSAV, PCA y defectos multivalvulares en el síndrome Edwards; aorta bicúspide, coartación de aorta y estenosis aórtica en el síndrome de Turner, y estenosis aórtica supravalvar y estenosis pulmonar en el síndrome Williams.

Palabras clave: Cardiopatías congénitas; Síndrome de Down; Síndrome de la trisomía 13; Síndrome de la trisomía 18; Síndrome de Turner; Síndrome de Williams (fuente: DeCS BIREME).

\section{Congenital heart disease associated with the most prevalent chromosomal syndromes: a literature review}

Most frequent chromosomal syndromes like Down, Patau, Edwards, Turner, and Williams affect the pediatric population in various ways, and congenital heart disease explains the altered quality of life they suffer. There is a lack of studies reviewing the cardiac anomalies in these syndromes, and the ones that exist are publications from past decades. We reviewed databases such as MEDLINE, LILACS, SCIELO, and Google Scholar, selecting the best possible evidence, and each chromosomal syndrome was investigated in relation to congenital heart disease, constituting five search groups. The article shows the characteristics of each heart disease described in the studies reviewed, the author, date of publication, country, and population studied, as well as a brief description of the frequency of the disease and its mortality. The results described in this review were contrasted with previous existing literature to verify if there was correspondence between the reported frequencies. The most frequent congenital heart diseases were atrioventricular septal defect (AVSD), ventricular septal defect (VSD), atrial septal defect (ASD), and persistent ductus arteriosus (PDA) in Down syndrome patients, PDA, ASD, and VSD in Patau syndrome patients, AVSD, PDA and valvular defects in Edwards syndrome, bicuspid aortic valve, aortic coarctation and aortic stenosis in Turner syndrome, and supravalvular aortic stenosis and pulmonary stenosis in Williams syndrome.

Keywords: Heart defects congenital; Down syndrome; Trisomy 13 syndrome; Trisomy 18 syndrome; Turner Syndrome; Williams Syndrome (source: MeSH NLM). 


\section{Introducción}

Los síndromes cromosómicos hacen referencia a cualquier alteración en el número o estructura normales de los cromosomas, entre ellas tenemos a las aneuploidías, caracterizadas por la pérdida o ganancia de material genético dando como resultado un número anormal de cromosomas, y las deleciones, que son defectos estructurales consecuencia de la pérdida de un número variable de genes. Estas alteraciones cromosómicas predisponen a aquellas personas que los presentan a padecer enfermedades congénitas, siendo las más comunes las cardiopatías congénitas ${ }^{(1)}$.

Las malformaciones cardiacas, término usado para definir las anormalidades en el corazón y sus grandes vasos, consecuencia de un fallo en su embriogénesis, se detectan en 3-5\% de los recién nacidos y 1 de cada 33 cursa con una anomalía grave ${ }^{(1,2)}$. Los avances tecnológicos en la genética han permitido esclarecer mejor el rol de las alteraciones cromosómicas en la génesis de las cardiopatías congénitas y su asociación con algún síndrome.

La presente revisión se centrará en la evidencia actual de las cardiopatías congénitas asociadas a síndromes cromosómicos frecuentes, debido al rol trascendente que tiene la evolución natural de este tipo de patologías en el desarrollo de los niños. Esto, con el fin de dar al profesional de salud información relevante que apoye a la sospecha diagnóstica en pacientes con sintomatología cardíaca y que presenten un determinado tipo de síndrome, para que de esta manera se determine el tratamiento -clínico o quirúrgico- más adecuado, y así mejorar la calidad de vida de la persona afectada. El objetivo de la revisión es describir la frecuencia y analizar las características de las principales cardiopatías congénitas asociadas a síndromes cromosómicos más prevalentes en pacientes pediátricos.

\section{Metodología}

Se realizaron búsquedas en base de datos electrónicas como MEDLINE, LILACS, SCIELO, y Google Scholar. En el caso de MEDLINE se usaron términos MeSH y términos libres, en tanto que para las otras bases de datos se recurrieron a las palabras clave y uso de conectores con el fin de reducir los resultados a los más trascendentes. Los resultados de la búsqueda final se limitaron a artículos en inglés y español publicados entre los años 2000 y 2021 en seres humanos; las búsquedas iniciales se hicieron sin limitaciones para analizar si había algún artículo en otro idioma, pero que sea importante.

La selección de artículos por evaluar se realizó mediante la identificación de títulos y resúmenes que describían la frecuencia de cardiopatías congénitas asociadas a pacientes con anomalías cromosómicas, para lo cual se hizo una búsqueda individualizada por cada una de las anomalías cromosómicas que se tratarán en la presente revisión (síndrome Down, síndrome de trisomía 13, síndrome de trisomía 18, síndrome de Turner y síndrome de Williams), por ejemplo: "Cardiopatías congénitas asociadas a síndrome Down" (Figura 1).

\section{Resultados}

\section{Síndrome Down}

Es la anomalía cromosómica más frecuente, se da principalmente por tres mecanismos: trisomía 21 (95\%), translocación robertsoniana (3-4\%) y mosaicismo de trisomía 21 (1-2\%) ${ }^{(3)}$; en casi el $90 \%$ de casos de trisomía 21, el cromosoma 21 extra se origina en la madre, por tal motivo el riesgo aumenta con la edad materna avanzada. Según la OMS, se estima que la prevalencia global es de 10 en cada 10000 recién nacidos vivos, pero se hace hincapié en que las cifras dependen de variaciones socioculturales como la legalización del aborto y el diagnóstico prenatal precoz ${ }^{(4)}$.

Puede afectar a prácticamente todos los sistemas y órganos, pero entre las afecciones más prevalentes se hallan la dificultad para el aprendizaje, hipotiroidismo, cardiopatías congénitas, alteraciones a nivel gastrointestinal y leucemias. El diagnóstico es guiado por los criterios de Hall, evaluados en todo recién nacido vivo y se confirma por citogenética ${ }^{(4)}$. Es la trisomía con mejor tasa de supervivencia, de hecho, un estudio de cohorte retrospectiva evalúo 16506 nacidos vivos entre los años 19822003, encontrando que las tasas de supervivencia al mes, 1, 5 y 20 años fueron de $98 \%, 93 \%, 91 \%$ y $88 \%$, respectivamente ${ }^{(5)}$.

Se reportaron morbilidades asociadas como hipertrofia adenoidea, asma, hernia umbilical, e hipotiroidismo (6), la complicación más frecuente fue la hipertensión pulmonar, casi el $50 \%$ lo padece y se presenta más en asociación al defecto del tabique auriculoventricular; sin embargo, la literatura enfatiza en que bebés no afectados inicialmente con hipertensión pulmonar pueden volverse sintomáticos en la infancia o luego; asimismo, también se presentaron insuficiencia mitral, infecciones respiratorias y edema pulmonar ${ }^{(7)}$.

Aproximadamente $50 \%$ de niños con síndrome Down padece de algún tipo de cardiopatía congénita y la mayor mortalidad se halla en los dos primeros años ${ }^{(8)}$. En un estudio retrospectivo realizado en Marruecos se calculó que la tasa de mortalidad fue $14,1 \%{ }^{(8)}$, esto nos muestra un gran contraste con Suecia, en donde la tasa de mortalidad ha disminuido de 41 a $4 \%$ en un período de 50 años desde 1973 hasta $2003^{(9)}$; en Latinoamérica, Panamá, reporta una mortalidad de 3,45\% (6). 


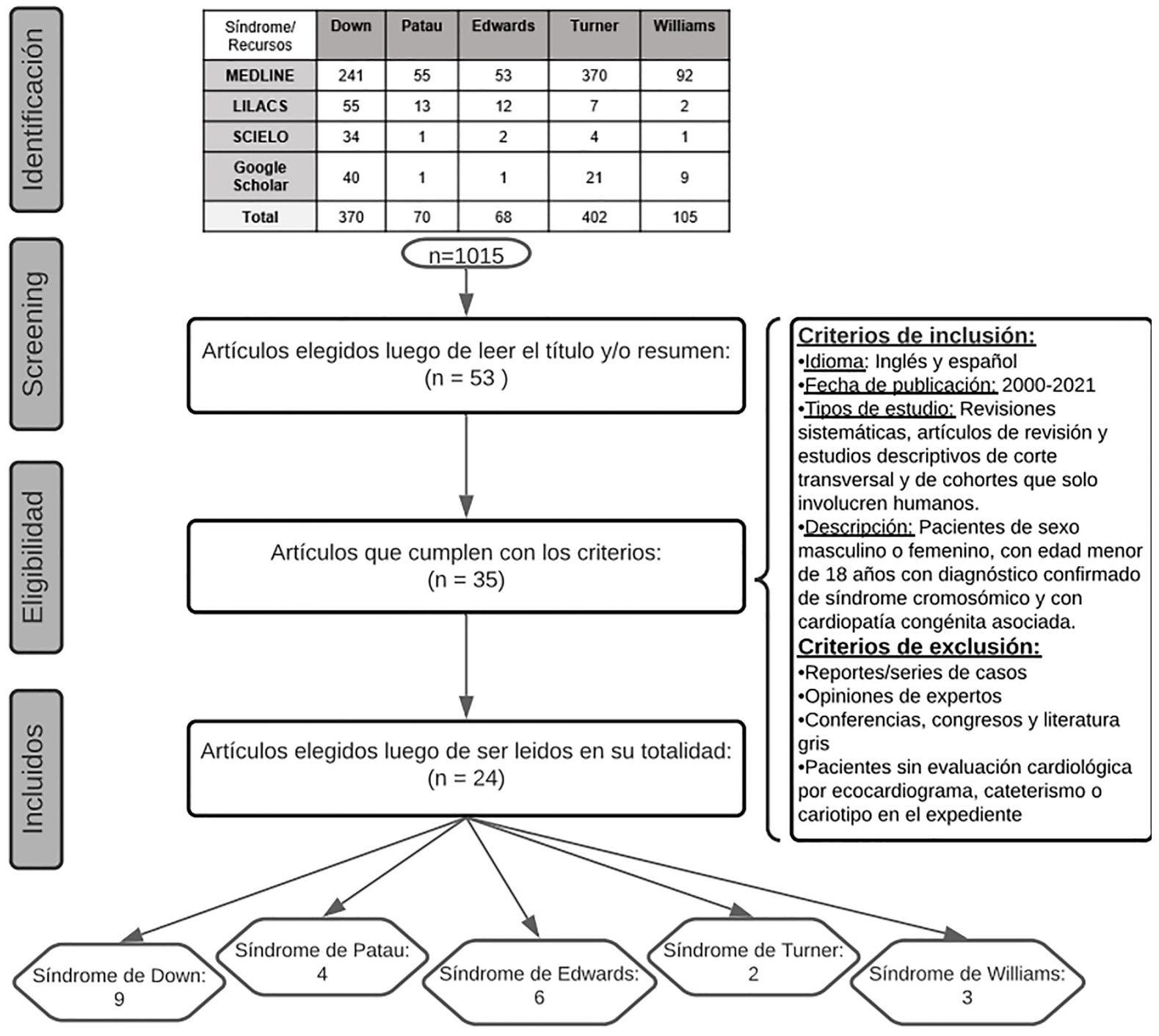

Figura 1. Modelo PRISMA

Además, un gran estudio poblacional en Inglaterra halló que la mortalidad se reducía de 30 a 5\% luego de una intervención quirúrgica temprana ${ }^{(10)}$. Las principales causas de mortalidad son choque séptico y cardiogénico ${ }^{(11,12)}$, por lo que se justifica que los pacientes con Síndrome Down tengan investigación en el período neonatal y postnatal, con la finalidad de reducir la morbilidad y mortalidad en relación con las complicaciones ${ }^{(13,14)}$. En la Tabla 1 se describen las principales cardiopatías congénitas frecuentemente asociadas con el síndrome Down.

\section{Síndrome Patau}

Este síndrome cursa con tres mecanismos patogénicos: trisomía 13, translocación robertsoniana y mosaicismo. La tríada clínica característica se constituye por micro/anoftalmia, paladar hendido y polidactilia posaxial ${ }^{(15)}$. Generalmente padecen de múltiples alteraciones ya sean anatómicas o funcionales, siendo el riesgo de muerte fetal del $80 \%$, pues se asocian malformaciones craneofaciales, oculares, cerebrales, hematológicas, abdominales y cardiopatías, debido a esto el pronóstico no es muy bueno, siendo la supervivencia menor a 1 año en su mayoría y sobrepasar los 10 años es excepcional ${ }^{(16)}$. La prevalencia es variable entre 1 de 10000 nacidos vivos o 1 de 30000 nacidos vivos. Es la tercera trisomía en frecuencia luego de la trisomía 21 y 18, dependiente de factores socioculturales ${ }^{(16)}$.

Las cardiopatías de la trisomía 13 con frecuencia son múltiples, es raro que se hallen aisladas y que solo una cardiopatía congénita afecte al paciente pediátrico. El diagnóstico se sospecha con ecografía del primer trimestre de embarazo más 
Tabla 1. Frecuencias de cardiopatías congénitas en síndrome Down

\begin{tabular}{|c|c|c|c|c|c|c|c|c|c|}
\hline $\begin{array}{l}\text { Autor } \\
\text { (fecha) }\end{array}$ & $\begin{array}{c}\text { De Rubens } \\
\text { et al. } \\
(2003)\end{array}$ & $\begin{array}{l}\text { Freeman } \\
\text { et al. } \\
(2008)\end{array}$ & $\begin{array}{l}\text { Irving } \\
\text { et al. } \\
(2012)\end{array}$ & $\begin{array}{c}\text { Bermúdez } \\
\text { et al. } \\
\text { (2015) }\end{array}$ & $\begin{array}{c}\text { Benhaourech } \\
\text { et al. } \\
(2016)\end{array}$ & $\begin{array}{c}\text { Bergstrom } \\
\text { et al. } \\
(2016)\end{array}$ & $\begin{array}{c}\text { Ruz } \\
\text { et al. } \\
(2017)\end{array}$ & $\begin{array}{c}\text { Yaqoob } \\
\text { et al. } \\
(2019)\end{array}$ & $\begin{array}{l}\text { Sotillo } \\
\text { et al. } \\
(2020)\end{array}$ \\
\hline $\begin{array}{l}\text { Localización } \\
\text { de estudio }\end{array}$ & $\begin{array}{l}\text { México } \\
\text { (México) }\end{array}$ & $\begin{array}{c}\text { Multicéntrico } \\
\text { (USA) }\end{array}$ & $\begin{array}{l}\text { Newcastle } \\
\text { (Inglaterra) }\end{array}$ & $\begin{array}{l}\text { Paraná } \\
\text { (Brasil) }\end{array}$ & $\begin{array}{l}\text { Casablanca } \\
\text { (Marruecos) }\end{array}$ & $\begin{array}{c}\text { Multicéntrico } \\
\text { (Suecia) }\end{array}$ & $\begin{array}{l}\text { Medellín } \\
\text { (Colombia) }\end{array}$ & $\begin{array}{l}\text { Multicéntrico } \\
\text { (Pakistán) }\end{array}$ & Panamá \\
\hline $\begin{array}{l}\text { Período de } \\
\text { estudio }\end{array}$ & 1994-1998 & 2000-2004 & $1985-2006$ & $2005-2013$ & $2008-2014$ & $1992-2012$ & $2010-2013$ & $2010-2016$ & 2011-2018 \\
\hline Población & $270(160)$ & $1673(1469)$ & $821(342)$ & $1207(604)$ & 128 & $2588(1387)$ & 99 & $350(117)$ & $67(53)$ \\
\hline DSAV & $9 \% *$ & $17,2 \% *$ & $43 \% *$ & $42,1 \% *$ & $29 \% *$ & $42 \% *$ & $31,31 \%$ & $12 \% *$ & $13,79 \%^{*}$ \\
\hline CIV & $30 \% *$ & $19,2 \% *$ & $31 \% *$ & $12,7 \% *$ & $21.5 \% *$ & $22,1 \% *$ & $61,62 \%$ & $41 \% *$ & $31,03 \% *$ \\
\hline $\mathrm{CIA}$ & $38 \% *$ & $18,6 \% *$ & $15 \% *$ & $14,6 \% *$ & $19.9 \% *$ & $16,2 \% *$ & $46,46 \%$ & $8,5 \% *$ & $6,9 \%^{*}$ \\
\hline TOF & $1 \% *$ & $2,7 \% *$ & $5 \% *$ & $2 \% *$ & $5.4 \% *$ & $2,6 \% *$ & $17,17 \%$ & $5,1 \% *$ & $3,45 \% *$ \\
\hline PCA & $21 \% *$ & $<1,3 \% *$ & $4 \% *$ & $6,6 \% *$ & $16.7 \% *$ & $5 \% *$ & $38,38 \%$ & $15,4 \% *$ & $20,69 \% *$ \\
\hline
\end{tabular}

DSAV. Defecto septal auriculoventricular. CIV. Comunicación interventricular. CIA. Comunicación interauricular. TOF. Tetralogía de Fallot. PCA. Persistencia de conducto arterioso. $\left(^{*}\right)$ Diagnóstico principal.

el uso de marcadores cromosómicos, y en el segundo trimestre con estudio morfológico, en tanto, la confirmación se da con el cariotipo ${ }^{(16)}$. Las cardiopatías congénitas se presentan en el $80 \%$ de casos, la mediana de supervivencia es de 7 días y la mayoría fallece en el primer mes de vida ${ }^{(17)}$. En un estudio se halló que la menor frecuencia de anomalías cardíacas contribuye a una supervivencia más prolongada ${ }^{(18)}$.

La principal causa de óbito son las complicaciones cardiopulmonares, el 50\% fallece el primer mes de nacido y el $70 \%$ a los 6 meses; sin embargo, la mortalidad intrahospitalaria disminuye en $45 \%$ en pacientes que se someten a intervención quirúrgica ${ }^{(19)}$. Por ello, si bien la mayoría de pacientes fallecen en las primeras semanas luego del nacimiento, algunos pueden sobrevivir más allá del primer año ${ }^{(20,21)}$. En la literatura revisada se encontraron varias cardiopatías congénitas frecuentemente asociadas con síndrome Patau (Tabla 2).

\section{Síndrome Edwards}

Es un desorden genético causado por la presencia, ya sea total o parcial, de un cromosoma 18 adicional, este síndrome es la segunda trisomía más frecuente después del síndrome Down. Su prevalencia global se encuentra entre 1 de 7000 nacidos vivos, la cual aumenta conforme la edad materna sea mayor. La tasa de mortalidad después del nacimiento es elevada, con un porcentaje del $50 \%$ que sobrevive después de la primera semana y un $5-10 \%$ que logra superar el año de edad ${ }^{(22)}$. El diagnóstico de estos pacientes se realiza, en su mayoría, durante la etapa prenatal por la presencia de malformaciones anatómicas en el ultrasonido (translucencia nucal, retardo en el crecimiento) o por el cariotipo después de una amniocentesis o cordocentesis ${ }^{(22)}$.

Las manifestaciones clínicas de este síndrome incluyen: alteraciones en el crecimiento, retraso psicomotor, cardiopatías

Tabla 2. Frecuencia de cardiopatías congénitas en el síndrome Patau

\begin{tabular}{lcccc}
\hline Autor (fecha) & $\begin{array}{c}\text { Lin et al. } \\
\text { (2007) }\end{array}$ & $\begin{array}{c}\text { Maeda et al. } \\
\mathbf{( 2 0 1 1 )}\end{array}$ & $\begin{array}{c}\text { Polli et al. } \\
\text { (2013) }\end{array}$ & $\begin{array}{c}\text { Kosiv et al. } \\
\text { (2017) }\end{array}$ \\
\hline Localización de estudio & Taipei (China) & Multicéntrico (Japón) & Porto Alegre (Brasil) & Multicéntrico (USA) \\
Período de estudio & $1985-2004$ & $2005-2008$ & $2000-2004$ & $2004-2015$ \\
Población & 22 & 23 & 15 & 555 \\
Atresia-Estenosis pulmonar & $18 \%$ & - & $16 \%$ & $11 \%$ \\
VD de doble salida & $<15 \%$ & $11 \%^{*}$ & - & $12 \%$ \\
PCA & $68 \%$ & - & $37 \%$ & $52 \%$ \\
CIA & $50 \%$ & $19 \%^{*}$ & $53 \%$ & $45 \%$ \\
CIV & $50 \%$ & $15 \%^{*}$ & $26 \%$ & $40 \%$ \\
$\begin{array}{l}\text { PCA. Persistencia de conducto arterioso. CIA. Comunicación interauricular. CIV. Comunicación interventricular. } \\
\text { (*) Diagnóstico principal. }\end{array}$ & &
\end{tabular}


Tabla 3. Frecuencia de cardiopatías congénitas en el síndrome Edwards

\begin{tabular}{|c|c|c|c|c|c|c|}
\hline Autor (fecha) & $\begin{array}{l}\text { Lin et al. } \\
\text { (2006) }\end{array}$ & $\begin{array}{l}\text { Kosho et al. } \\
\quad(2006)\end{array}$ & $\begin{array}{l}\text { Kaneko et al. } \\
\text { (2008) }\end{array}$ & $\begin{array}{l}\text { Peterson et al. } \\
\text { (2017) }\end{array}$ & $\begin{array}{l}\text { Kosiv et al. } \\
\text { (2017) }\end{array}$ & $\begin{array}{l}\text { Cooper et al. } \\
\text { (2019) }\end{array}$ \\
\hline Localización de estudio & $\begin{array}{l}\text { Taipei } \\
\text { (Taiwan) }\end{array}$ & $\begin{array}{l}\text { Nagano } \\
\text { (Japón) }\end{array}$ & $\begin{array}{l}\text { Tokio } \\
\text { (Japón) }\end{array}$ & $\begin{array}{l}\text { Multicéntrico } \\
\text { (USA/Canadá) }\end{array}$ & $\begin{array}{l}\text { Multicéntrico } \\
\text { (USA) }\end{array}$ & $\begin{array}{l}\text { Multicéntrico } \\
\text { (USA) }\end{array}$ \\
\hline Período de estudio & 1988-2004 & 1994-2003 & 2000-2005 & $1982-2008$ & 2004-2015 & 2010-2017 \\
\hline Población & $39(31)$ & $24(24)$ & $22(22)$ & $121(69)$ & $1020(925)$ & $270(270)$ \\
\hline CIV & $94 \%$ & $75 \%$ & $77,2 \%$ & $48 \% *$ & $60 \%$ & $54,1 \%$ * \\
\hline PCA & $77 \%$ & $75 \%$ & $68,1 \%$ & $5 \% *$ & $57 \%$ & $7,4 \% *$ \\
\hline $\mathrm{ClA}$ & $68 \%$ & $21 \%$ & - & - & $43 \%$ & $3,3 \% *$ \\
\hline $\mathrm{COA}$ & $<6 \%$ & $8,3 \%$ & $27,2 \%$ & $9 \% *$ & $13 \%$ & $3 \% *$ \\
\hline TOF & $<6 \%$ & $8,3 \%$ & $4,5 \%$ & $14,8 \%$ * & $6 \%$ & $5,9 \% *$ \\
\hline Estenosis aórtica & $10 \%$ & - & - & - & $16 \%$ & - \\
\hline Insuficiencia aórtica & & - & - & - & & - \\
\hline AoV Atresia & $<6 \%$ & - & - & - & & - \\
\hline
\end{tabular}

CIV. Comunicación interventricular. PCA. Persistencia de conducto arterioso. CIA. Comunicación interauricular. CoA. Coartación de aorta. TOF. Tetralogía de Fallot. AoV atresia. Atresia de válvula aórtica. $\left({ }^{*}\right)$ Diagnóstico principal.

congénitas, anomalías faciales, malformaciones del cráneo, el tórax, el abdomen y genitales ${ }^{(23)}$. Siendo los defectos cardíacos congénitos las manifestaciones clínicas más frecuentes (90\% de los casos), además de ser la principal causa de muerte ${ }^{(24)}$. Entre las más frecuentes tenemos: defectos del septo, ductus arterioso persistente y defectos valvulares ${ }^{(19)}$ (Tabla 3).

\section{Síndrome Turner}

También llamado síndrome de Ullrich-Turner o monosomía X; como su propio nombre lo indica, es causado por la ausencia parcial o total del segundo cromosoma $X$ siendo la única monosomía compatible con la vida. La gran mayoría de gestaciones $« 45, X »$ concluyen en abortos espontáneos, teniendo como principal causa las anormalidades cardíacas (25,26). Se estima que el síndrome Turner está presente en el 3\% de fetos femeninos, de los cuales un 10\% sobrevive; asimismo, tiene una prevalencia de 1 de 2500 nacidas vivas. No se encontraron datos de mortalidad posnatal, salvo por un estudio prospectivo realizado con 156 mujeres en 1986, seguidas por 17 años, con 15 de ellas fallecidas en este transcurso $(3,6 \%)^{(27)}$.

El diagnóstico se realiza mediante la identificación de características clínicas reconocibles y el cariotipo ${ }^{(26)}$. Este síndrome se caracteriza por la baja estatura, retraso intelectual, falla ovulatoria prematura, anomalías faciales, cuello alado, linfedema y defectos cardíacos congénitos. El fenotipo que pueden presentar es muy variable entre cada paciente.
El porcentaje de mujeres que presentan cardiopatías congénitas varía según distintas bibliografías (25-50\%) siendo las más frecuentes aquellas que afectan al corazón izquierdo: la válvula aórtica bicúspide, coartación de aorta y estenosis valvular aórtica ${ }^{(26,27)}$. En la Tabla 4 se detalla la literatura revisada con las principales cardiopatías congénitas asociadas al síndrome Turner.

\section{Síndrome Williams}

Este síndrome también conocido como síndrome de WilliamsBeuren, es causado por la pérdida de 26 a 28 genes debido a la deleción del cromosoma 7, específicamente 7q11.23. La prevalencia de esta afección se encuentra en 1 de 10000 nacidos vivos. Producto de esta anormalidad genética se presentan alteraciones que involucran distintos sistemas, entre ellos: nervioso, hematológico, tejido conectivo, osteoarticular, cardiovascular, entre otros ${ }^{(28)}$. El compromiso cardiovascular está dado por la afectación del gen que codifica a la elastina (ELN) lo que conlleva a un depósito anormal o deficiente de esta proteína durante el periodo embrionario, muy importante para la formación de los vasos sanguíneos. Las malformaciones congénitas que atacan al sistema cardiovascular están presentes en cerca de un $75-80 \%$ de los pacientes ${ }^{(29)}$.

En la bibliografía revisada, las alteraciones vasculares como la estenosis de la arteria pulmonar y la estenosis aórtica supravalvar fueron los defectos más frecuentes a comparación de las alteraciones intracardiacas que eran más catalogadas como hallazgos atípicos ${ }^{(28-30)}$. En la literatura revisada se encontraron 
Tabla 4. Frecuencia de cardiopatías congénitas en el síndrome Turner

\begin{tabular}{|c|c|c|}
\hline Autor (fecha) & $\begin{array}{c}\text { Volki et al. } \\
(2005)\end{array}$ & $\begin{array}{c}\text { Kim et al. } \\
(2010)\end{array}$ \\
\hline Localización de estudio & $\begin{array}{c}\text { Erlangen } \\
\text { (Alemania) }\end{array}$ & $\begin{array}{l}\text { Cincinati } \\
\text { (USA) }\end{array}$ \\
\hline Período de estudio & 1980-2003 & $2007-2009$ \\
\hline Población & $117(35)$ & $51(51)$ \\
\hline CoA & $15,4 \%$ & $15,7 \%$ \\
\hline Válvula aórtica bicúspide & $12,8 \%$ & $39,2 \%$ \\
\hline Estenosis valvular aórtica & $8,5 \%$ & - \\
\hline CIV & $4,3 \%$ & - \\
\hline PAPVD & $3,4 \%$ & $15,7 \%$ \\
\hline $\mathrm{CIA}$ & $1,7 \%$ & - \\
\hline
\end{tabular}

CoA. Coartación de aorta. CIV. Comunicación interventricular. PAPVD. Drenaje venoso pulmonar anómalo parcial. CIA. Comunicación interauricular.

varias cardiopatías congénitas asociadas al síndrome Williams (Tabla 5).

En resumen, y según lo revisado en la literatura, las cardiopatías congénitas más frecuentes asociadas a síndromes cromosómicos se describen en la Tabla 6.

\section{Discusión}

\section{Síndrome Down}

Si bien la cardiopatía congénita más asociada al síndrome Down fue el defecto en el tabique AV, hubo gran variabilidad en el tamaño de las muestras y el porcentaje obtenido, siendo los más elevados en Inglaterra, Brasil y Suecia, en contraste con Panamá, Pakistán y México, el cual según su población estudiada solo estuvo presente en $6 \%$ de la muestra.

La comunicación interventricular fue más frecuente en Colombia y Pakistán, en donde el 62 y $41 \%$ de sus muestras padecían este tipo de cardiopatía congénita, en las demás poblaciones de estudio no se halló mayor variación, y la frecuencia se hallaba entre 13-31\%. Colombia también obtuvo los mayores índices de comunicación interauricular.

La tetralogía de Fallot fue una cardiopatía bastante infrecuente, los estudios obtuvieron una variación en el rango

Tabla 5. Frecuencia de cardiopatías congénitas en el síndrome Williams

\begin{tabular}{|c|c|c|c|}
\hline Autor (fecha) & $\begin{array}{l}\text { Bruno et al. } \\
(2003)\end{array}$ & $\begin{array}{c}\text { Del Pasqua et al. } \\
\qquad(2009)\end{array}$ & $\begin{array}{c}\text { Collins et al. } \\
\qquad(2007)\end{array}$ \\
\hline Localización de estudio & $\begin{array}{c}\text { Córdoba } \\
\text { (Argentina) }\end{array}$ & $\begin{array}{l}\text { Roma } \\
\text { (Italia) }\end{array}$ & $\begin{array}{l}\text { Filadelfia } \\
\text { (USA) }\end{array}$ \\
\hline Período de estudio & $1980-2002$ & $1981-2006$ & $1980-2007$ \\
\hline Población & $53(85)$ & $150(113)$ & $129(129)$ \\
\hline Estenosis aórtica supravalvar & $71 \%$ & $64,6 \%$ & $57 \%$ \\
\hline Estenosis pulmonar periférica & \multirow{2}{*}{$38 \%$} & \multirow{3}{*}{$45,1 \%$} & $62 \%$ \\
\hline Estenosis pulmonar supravalvar & & & $20 \%$ \\
\hline Estenosis pulmonar valvular & $11 \%$ & & $16 \%$ \\
\hline Prolapso válvula mitral & $27 \%$ & $6,2 \%$ & $15 \%$ \\
\hline CoA & $4 \%$ & $6,2 \%$ & $18 \%$ \\
\hline CIV & $4 \%$ & $7,9 \%$ & $21 \%$ \\
\hline
\end{tabular}

CoA. Coartación de aorta. CIV. Comunicación interventricular 
Tabla 6. Cardiopatías congénitas más frecuentes por síndrome

\begin{tabular}{lccrr}
\hline $\begin{array}{l}\text { Síndrome } \\
\text { Down }\end{array}$ & Síndrome Patau & Síndrome Edwards & Síndrome Turner & Síndrome Williams \\
\hline DSAV (9-43\%) & PCA (37-68\%) & CIV (48-94\%) & $\begin{array}{r}\text { Válvula aórtica bicúspide } \\
(21.8-39.2 \%)\end{array}$ & $\begin{array}{c}\text { Estenosis aórtica supravalvar } \\
(57-71 \%)\end{array}$ \\
CIV (12.7-41\%) & CIA (19-53\%) & PCA (57-77\%) & CoA (15.4-15.7\%) & Estenosis pulmonar \\
CIA (6.9-38\%) & CIV (15-50\%) & CIA (21-68\%) & Estenosis valvular aórtica (8.5\%) & Prolapso válvula mitral (6.2-27\%) \\
PCA (1.3-21\%) & TF (5-23\%) & PAPVD & CIV (4-21\%) \\
TF (1-5.4\%) & Estenosis Pulmonar (11-18\%) & Estenosis aórtica (10-16\%) & CIV (4.3\%) & CIA (4-18\%) \\
\hline
\end{tabular}

DSAV. Defecto septal auriculoventricular. CIV. Comunicación interventricular. CIA. Comunicación interauricular. TF. Tetralogía de Fallot. PCA. Persistencia de conducto arterioso. CoA. Coartación de aorta.. PAPVD. Drenaje venoso pulmonar anómalo parcial.

de 1-5,5\%, a excepción de Colombia en donde llegó a 17,17\%. Por otro lado, la persistencia del conducto arterioso fue más frecuente en Colombia, México, Panamá, Marruecos y Pakistán; los otros estudios descriptivos de países como Estados Unidos, Inglaterra, Suecia y Brasil tuvieron una frecuencia de entre 1-7\%.

La explicación sobre la gran variabilidad de datos expresados como porcentajes son la variación sociocultural y la mayor capacidad de diagnóstico de cardiopatías congénitas con el paso de los años; asimismo, la gran proporción en el estudio de Colombia puede hallar su explicación parcial en que sus porcentajes no excluyeron datos de cardiopatías múltiples, a diferencia de los demás estudios, en donde se consideró solo el recuento de cardiopatías aisladas.

Si bien las cardiopatías congénitas se diagnostican hasta en 50\% de pacientes con síndrome Down, la frecuencia es menor en aquellos con mosaicismo por trisomía $21^{(1)}$. El hecho es que los defectos cardiacos congénitos se han vuelto menos comunes en bebés diagnosticados con síndrome Down, probablemente por mejoras en el diagnóstico prenatal y aborto selectivo ${ }^{(9)}$.

\section{Síndrome Patau}

A pesar de los pocos estudios obtenidos y de la muestra reducida de estos, la cardiopatía más asociada en China y Estados Unidos fue la persistencia del ductus arterioso, a diferencia de Brasil y Japón, en donde los primeros lugares lo ocupan los defectos en el tabique interauricular e interventricular. La atresia-estenosis pulmonar tuvo un rango de entre $11-18 \%$, pero en el estudio de Japón la frecuencia fue nula.

La tetralogía de Fallot fue más frecuente en China en comparación con los demás estudios descriptivos; por otro lado, el ventrículo derecho de doble salida y la coartación de aorta obtuvieron frecuencias similares en China, Japón y Estados
Unidos, cabe reiterar que en Brasil no se obtuvieron datos sobre estas cardiopatías.

Se comparte la explicación brindada para el síndrome Down, además, es importante detallar que en el estudio de Japón se consideraron porcentajes de cardiopatías congénitas aisladas y no en forma de múltiples como en los estudios de China, Brasil y Estados Unidos.

\section{Síndrome Edwards}

Se presenta a la comunicación interventricular como la cardiopatía congénita más frecuente (48-94\%) en todos los estudios revisados, siendo también la más frecuente reportada por las bibliografías revisadas ${ }^{(2)}$ donde se la presenta con una frecuencia mayor al 90\%, seguido por la PCA, con muchas más variaciones entre cada uno de los estudios.

Se revisaron estudios que solo consideraban dentro de la estadística a los diagnósticos principales de cada caso, lo cual explicaría el porqué de variaciones muy pronunciadas en los porcentajes, además de subestimar la frecuencia de otras patologías que podrían estar presentes concomitantemente en cada uno de los pacientes evaluados. Esto dificulta la comparación de los datos y conocer los valores absolutos de cada una de ellas.

Existieron estudios que no reportaron la presencia de algunas cardiopatías, como en el caso de estenosis aórtica, insuficiencia aórtica y atresia de la válvula aórtica, lo que puede ser explicado por la diferencia en el número de poblaciones evaluadas, puesto que algunos estudios presentaban 22 casos revisados frente a otro con 1020 pacientes estudiados.

\section{Síndrome Turner}

Las dos cardiopatías congénitas más frecuentes en el síndrome Turner observadas en los dos estudios revisados fueron aquellas 
que comprometen a la arteria y válvula aórticas: coartación de aorta y válvula aórtica bicúspide. Esto se correlaciona con lo descrito por Bondy ${ }^{(27)}$ que hizo una revisión a cinco estudios con más de cien pacientes, con fechas de publicación anteriores a nuestra búsqueda, en los cuales se determinó que la válvula aórtica bicúspide (12-30\%) y la coartación de aorta (7-18\%) son las presentaciones más frecuentes, seguidas por defectos septales y circulación pulmonar venosa anómala parcial. Sin embargo, Lin ${ }^{(2)}$ describe otras anomalías que presentan una frecuencia similar a las mencionadas: elongación de aorta transversal (25-50\%), dilatación aórtica (25-40\%) y anomalías coronarias congénitas (5-25\%).

La frecuencia de coartación de aorta coincide en los estudios que se analizaron ${ }^{(26,27)}$, pero no hubo una correspondencia en los porcentajes de las demás cardiopatías e, incluso, no se reportó la presencia de otras como la estenosis valvular aórtica o las comunicaciones intercavidades. La poca cantidad de estudios encontrados y la diferencia entre el número de población no permitiríanunabuenacomparaciónentrelos resultadosencontrados. Esto agregado a que no se reportaron otras cardiopatías congénitas que sí fueron consideradas en el estudio de Volkl ${ }^{(25)}$.

\section{Síndrome Williams}

Los tres estudios revisados señalan a la estenosis aórtica supravalvar como la manifestación anómala cardíaca más frecuente en este síndrome. La literatura científica también la presenta como la principal, con frecuencias que van desde 45 al $75 \%{ }^{(28)}$. Lo mismo sucede con la estenosis pulmonar en sus diferentes presentaciones (periférica, supravalvar, valvular) con frecuencias que la sitúan en un rango de $37-75 \%$, con la mayoría de estudios que reportan una frecuencia del $40 \%{ }^{(29)}$.

El prolapso de válvula mitral representa el $27 \%$ de casos en Argentina, frecuencia un poco mayor en comparación con los Estados Unidos de Norteamérica (EUA) (15\%) e Italia (6,2\%). La coartación de aorta y la comunicación interventricular tuvieron similar frecuencia en Argentina e Italia, pero en EUA el porcentaje aumentó. Por todo lo mencionado se puede inferir que las poblaciones de Argentina e Italia son similares en cuanto a cardiopatías congénitas asociadas a síndrome de Williams, y en su mayoría los porcentajes se elevan en el caso de EUA, en donde es más frecuente la estenosis pulmonar periférica.

\section{Limitaciones}

Los estudios revisados, en su mayoría, tienen fechas de publicación de la década pasada lo cual dificulta conocer la actualidad de estas cardiopatías. Existe una cantidad limitada de estudios que tratan sobre cardiopatías asociadas a síndromes cromosómicos diferentes al síndrome Down; además, contar con un número de población reducida no permite tener una aproximación tan exacta a la frecuencia real de estas patologías. La nacionalidad, los tipos de estudios (algunos multicéntricos), metodología, la forma de presentación de la información y resultados son muy variados, esto dificulta la posibilidad de realizar una comparación y posterior análisis de los mismos. Finalmente, la ausencia de publicación de artículos nacionales de este tipo no permite conocer información relevante sobre la situación actual del país, por ello la necesidad de realizar investigación científica sobre estos temas.

\section{Conclusiones}

La frecuencia de cardiopatías congénitas en niños con síndromes cromosómicos es alta, pero muy variable, por tal motivo, se recalca la importancia de investigación en el período neonatal, para así disminuir las tasas de morbilidad y mortalidad, y asegurar una mejor calidad de vida en el desarrollo de estos niños.

\section{Contribución de los autores}

JECL y OEEA: diseño de estudio, extracción y análisis de datos, redacción del manuscrito. GPHB: diseño de estudio, revisión del manuscrito y aprobación de la versión final del manuscrito.

\section{Referencias bibliográficas}

1. Zaidi S, Brueckner M. Genetics and Genomics of Congenital Heart Disease. Circulation Research. 2017;120(6):923-940. DOI: 10.1161/ CIRCRESAHA.116.309140

2. Lin A, Santoro S, High F, Goldenberg P, Gutmark-Little I. Congenital heart defects associated with aneuploidy syndromes: New insights into familiar associations. American Journal of Medical Genetics Part C: Seminars in Medical Genetics. 2019;184(1):53-63. DOI: 10.1002/ ajmg.c. 31760

3. Jones KL. Smith's recognizable patterns of human malformation, 6 th ed, Elsevier Saunders, Philadelphia 2006.

4. Díaz Cuéllar S, Yokoyama Rebollar E, Del Castillo Ruiz V. Genómica del síndrome de Down. 2016:289-295.
5. Kucik JE, Shin M, Siffel C, Marengo L, Correa A. Trends in survival among children with Down syndrome in 10 regions of the United States. Pediatrics 2013; 131 :e27. DOI: 10.1542/peds.2012-1616

6. Sotillo-Lindo J, Barrantes I. Prevalencia y perfil de cardiopatías congénitas en pacientes con Síndrome de Down. Hospital de Especialidades Pediátricas Omar Torrijos Herrera. 2011-2018. Revista Pediátrica de Panamá. 2020;37-40. DOI: 10.37980/im.journal. rspp.20201695

7. Ruz-Montes M, Cañas-Arenas E, Lugo-Posada M, Mejía-Carmona M, Zapata-Arismendy M, Ortiz-Suárez L, et al. Cardiopatías congénitas más frecuentes en niños con síndrome de Down. Revista Colombiana de Cardiología. 2017;24(1):66-70. DOI: https://doi.org/10.1016/j. rccar.2016.06.014 
8. Benhaourech S, Drighil A, Hammiri A. Congenital heart disease and Down syndrome: various aspects of a confirmed association. Cardiovascular Journal of Africa. 2016;27(5):287-290. DOI: 10.5830/ CVJA-2016-019

9. Bergstrom S, Carr H, Petersson G, Stephansson O, Bonamy A, Dahlstrom A, et al. Trends in Congenital Heart Defects in Infants with Down Syndrome. PEDIATRICS. 2016;138(1). DOI: 10.1542/peds.20160123

10. Irving C, Chaudhari M. Cardiovascular abnormalities in Down's syndrome: spectrum, management and survival over 22 years. 2012: 326-330. DOI: 10.1136/adc.2010.210534

11. De Rubens J, Del Pozzo B, Pablos J, Calderón C, Castrejón R. Malformaciones cardíacas en los niños con síndrome de Down. Revista Española de Cardiología; 2003: 94-96. DOI: 10.1157/13051617

12. Freeman S, Bean L, Allen E, Tinker S, Locke A, Druschel C et al. Ethnicity, sex, and the incidence of congenital heart defects: a report from the National Down Syndrome Project. Genetics in Medicine. 2008;10(3):173-180. DOI: 10.1097/GIM.0b013e3181634867

13. Yaqoob M, Manzoor J, Hyder S, Sadiq M. Congenital heart disease and thyroid dysfunction in down syndrome reported at children's hospital, Lahore, Pakistan. The Turkish Journal of Pediatrics. 2019;61(6):915. DOI: 10.24953/turkjped.2019.06.013

14. Bermudez B, Medeiros S, Bermudez M, Novadzki I, Magdalena N. Down syndrome: Prevalence and distribution of congenital heart disease in Brazil. Sao Paulo Medical Journal. 2015;133(6):521-524. https://doi.org/10.1590/1516-3180.2015.00710108

15. Patau K, Smith DW, Therman E, Inhorn S, Wagner HP. Multiple congenital anomaly caused by an extra autosome. Lancet 1960; 1:790. DOI: 10.1016/s0140-6736(60)90676-0

16. Ribate M, Puisac B, Ramos F. Trisomía 13 (síndrome de Patau). Asociación Española de Pediatría. 2010;1:91-95.

17. Lakovschek IC, Streubel B, Ulm B. Natural outcome of trisomy 13, trisomy 18, and triploidy after prenatal diagnosis. Am J Med Genet A 2011; 155A:2626. DOI: 10.1002/ajmg.a.34284

18. Peroos S, Forsythe E, Pugh JH, Arthur-Farraj P, Hodes D. Longevity and Patau syndrome: what determines survival? BMJ Case Rep 2012; 2012. DOI: $10.1136 / \mathrm{bcr}-06-2011-4381$

19. Kosiv K, Gossett J, Bai S, Collins R. Congenital Heart Surgery on InHospital Mortality in Trisomy 13 and 18. Pediatrics. 2017;140(5):1-7. DOI: 10.1542/peds.2017-0772

20. Lin $\mathrm{H}$, Lin S, Chen $\mathrm{Y}, \mathrm{Hsu} \mathrm{C}, \mathrm{Kao} \mathrm{H}$, Chen M, et al. Clinical characteristics and survival of trisomy 13 in a medical center in Taiwan, 1985-2004.
Pediatrics International. 2007;49(3):380-386. DOI: 10.1111/j.1442200X.2007.02377x

21. Polli J, de P. Groff D, Petry P, Mattos V, Rosa R, Zen P, et al. Trisomy 13 (Patau syndrome) and congenital heart defects. American Journal of Medical Genetics Part A. 2013;164(1):272-275. DOI: 10.1002/ ajmg.a.36193

22. Maeda J, Yamagishi H, Furutani Y, Kamisago M, Waragai T, Oana $S$, et al. The impact of cardiac surgery in patients with trisomy 18 and trisomy 13 in Japan. American Journal of Medical Genetics Part A. 2011;155(11):2641-2646. DOI: 10.1002/ajmg.a.34285

23. Kosho T, Nakamura T, Kawame H, Baba A, Tamura M, Fukushima Y. Neonatal management of trisomy 18: Clinical details of 24 patients receiving intensive treatment. American Journal of Medical Genetics Part A. 2006;140A(9):937-944. DOI: 10.1002/ajmg.a.31175

24. Peterson J, Kochilas L, Catton K, Moller J, Setty S. Long-Term Outcomes of Children With Trisomy 13 and 18 After Congenital Heart Disease Interventions. The Annals of Thoracic Surgery. 2017;103(6):1941-1949. DOI: 10.1016/j.athoracsur.2017.02.068

25. Völkl T, Degenhardt K, Koch A, Simm D, Dörr H, Singer H. Cardiovascular anomalies in children and young adults with UllrichTurner syndrome-the erlangen experience. Clinical Cardiology. 2005;28(2):88-92. DOI: 10.1002/clc.4960280209

26. Kim H, Gottliebsen W, Hor K, Backeljauw P, Gutmark-Little I, Salisbury $\mathrm{S}$, et al. Cardiovascular Anomalies in Turner Syndrome: Spectrum, Prevalence, and Cardiac MRI Findings in a Pediatric and Young Adult Population. American Journal of Roentgenology. 2011;196(2):454460. DOI: $10.2214 /$ AJR.10.4973

27. Del Pasqua A, Rinelli G, Toscano A, lacobelli R, Digilio C, Marino B, et al. New Findings concerning Cardiovascular Manifestations emerging from Long-term Follow-up of 150 patients with the Williams-BeurenBeuren syndrome. Cardiology in the Young. 2009;19(6):563-567. DOI: $10.1017 /$ S1047951109990837

28. Bruno E, Rossi N, Thüer O, Córdoba R, Alday L. Cardiovascular findings, and clinical course, in patients with Williams syndrome. Cardiology in the Young. 2003;13(6):532-536. DOI: https://doi.org/10.1017/ S1047951103001124

29. Collins R, Kaplan P, Somes G, Rome J. Cardiovascular Abnormalities, Interventions, and Long-term Outcomes in Infantile Williams Syndrome. The Journal of Pediatrics. 2010;156(2):253-258. DOl: 10.1016/j.amjcard.2009.10.069

30. Bondy C. Congenital Cardiovascular Disease in Turner Syndrome. Congenital Heart Disease. 2008;3(1):2-15. DOI: 10.1111/j.17470803.2007.00163.x 